Chirurg 2020 $91: 743-754$

https://doi.org/10.1007/s00104-020-01179-7

Online publiziert: 29. April 2020

(C) Der/die Autor(en) 2020
Katharina Joechle ${ }^{1}$ Eleni Gkika ${ }^{2}$ - Anca-Ligia Grosu ${ }^{2}$ - Sven A. Lang • Stefan Fichtner-Feigl'

' Klinik für Allgemein- und Viszeralchirurgie, Universitätsklinikum Freiburg, Freiburg, Deutschland

${ }^{2}$ Klinik für Strahlenheilkunde, Universitätsklinikum Freiburg, Freiburg, Deutschland

\title{
Intraoperative Strahlentherapie - Indikationen und Optionen in der Viszeralchirurgie
}

\section{Hintergrund}

Chirurgie und Strahlentherapie werden in der Medizin schon sehr lange als ineinandergreifendes Therapiekonzept verstanden. Bereits 1969 wurde die Durchführung einer intraoperativen Strahlentherapie (IORT) von Abe aus Kyoto, Japan, erstmalig beschrieben [1, 2, 30] und bald weltweit als Ergänzung der bis zu diesem Zeitpunkt gängigen perioperativen Bestrahlung übernommen. Auch heute findet die IORT besonders in Kombination mit ausgeprägter Maximalchirurgie, perioperativer, perkutaner Bestrahlung und Chemotherapie als Teil eines multidisziplinären Therapiekonzeptes Anwendung [63]. Dabei wird durch die chirurgische Exposition des Tumors und des Tumorbetts eine hohe Präzision gewährleistet, welche es erlaubt, die Strahlendosis im Bereich des Tumors zu erhöhen (Dosiseskalation) und gleichzeitig gesundes Gewebe als den dosislimitierenden Faktor vor Strahlung zu schützen. Somit wird im Sinne einer zielgerichteten Therapie ein hoher therapeutischer Index bei geringen Nebenwirkungen erreicht [59]. Aus diesem Grund bietet die IORT besonders dann einen Vorteil, wenn die lokale Tumorkontrolle das Langzeitüberleben und die Funktionalität entscheidend beeinflusst [18], diese jedoch nicht allein durch eine chirurgische Resektion zu erreichen ist oder eine Strahlendosis erfordert, welche die Strahlentoleranz gesunden Gewebes im Rahmen einer perkutanen Bestrahlung übertreffen würde [59].
Diese Übersichtsarbeit soll eine umfassende und strukturierte Zusammenfassung über die Hauptindikationen der IORT bei intraabdominellen und retroperitonealen Tumoren geben und dabei die aktuelle Studienlage beleuchten.

\section{Techniken}

Zur Durchführung einer IORT wird in der Regel ein einziges Strahlenfeld in einer festgelegten Distanz zwischen Strahlenquelle und Zielvolumen genutzt [35]. Die chirurgische Exposition des Zielvolumens (Tumor bzw. Tumorbett) ist der wichtigste Schritt, tumorfreies Gewebe und funktionell relevante Strukturen wie Dünn- und Dickdarm, Gefäße und Nerven vor Strahlung zu schützen. Dabei ist besonders die intraoperative Interaktion und Absprache von Chirurgen und Strahlentherapeuten notwendig, sodass entsprechende Strukturen aus dem Strahlenfeld mobilisiert und durch eine temporäre Tamponade vor der Bestrahlung geschützt werden. Als besonders klinisch relevant und dosislimitierend zu sehen ist die Toxizität an Nerven, insbesondere am Plexus lumbosacralis bei Bestrahlung pelviner Tumoren, am Ureter oder dem Ductus choledochus. Da sich u.a. diese Strukturen in nur sehr geringem Maße mobilisieren lassen, können spezielle Strahlenschutzplatten zwischen zu bestrahlendem und zu schützendem Gewebe eingebracht werden. Diese bestehen in der Regel aus metallabsorbierendem Material wie z.B. Bleib. Entsprechend wurden in experimentellen und klini- schen Studien die Strahlentoleranz sowie die Strahlenbelastung gesunden Gewebes trotz Abschirmung beschrieben [27, 83]. Nach entsprechender Mobilisation, Tamponade und ggf. Schutz durch absorbierende Metallplatten können die mobilen Applikatoren so genau wie möglich platziert werden. Diesbezüglich kommen besonders Elektronen (IOERT), Hochdosisleistungbrachytherapie (HDR-IORT) und Röntgenstrahlen (kV-IORT) zum Einsatz.

Über mobile, lineare Beschleuniger (z. B. Novac7, Hitesys SPA, Aprillia, Italien; 7-10 MeV und Mobetron, IntraOp Medical Corporation, Sunnyvale, CA, USA; 4-12 MeV) lassen sich mittels der IOERT im Vergleich zur HDR-IORT oder kV-IORT höhere Eindringtiefen und homogenere Strahlendosen erreichen. Gleichzeitig sind diese Applikatoren jedoch sehr starr, sodass deren Anwendung z. B. im Becken oder in engen Körperhöhlen erschwert ist. Es wird typischerweise ein Energieniveau von $4-12 \mathrm{MeV}$ verwendet. Standard-(Rund-)Applikatoren haben einen Durchmesser von 3-4 cm bis $\mathrm{zu} 15 \mathrm{~cm}$ und die Strahlenfelder sind (je nach Hersteller) auf einen maximalen Durchmesser von $10-15 \mathrm{~cm}$ begrenzt. Die Verschreibung erfolgt typischerweise auf die $90 \%$-Isodose, wobei $100 \%$ der Dosis dem Dosismaximum entspricht $[9,35,63]$.

Die HDR-IORT-Brachytherapie mit Afterloading-Systemen hat den Vorteil eines sehr steilen Gradienten. Die IORT wird mit einem flexiblen Applikator (z. B. Harrison-Anderson-Mick [HAM] oder Freiburger Flab, Elekta AB, Stockholm, 


\section{Übersichten}

:

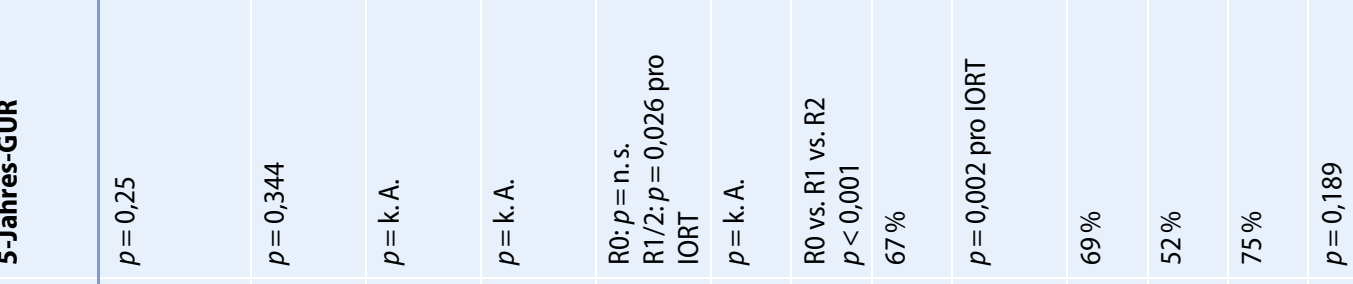

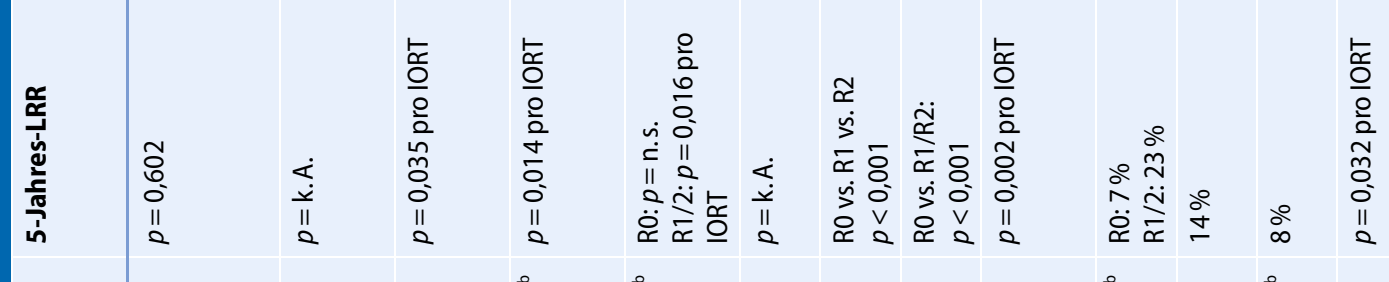

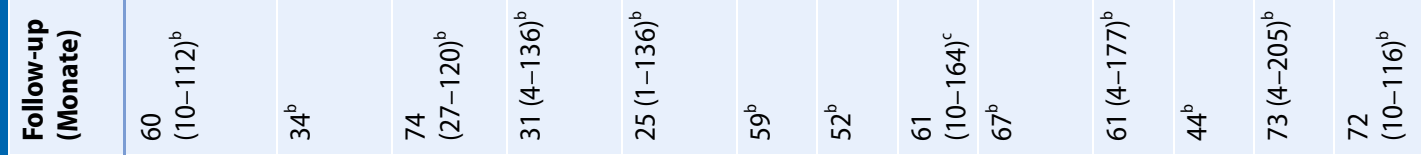

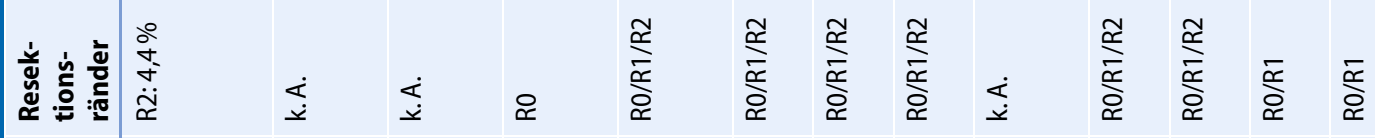

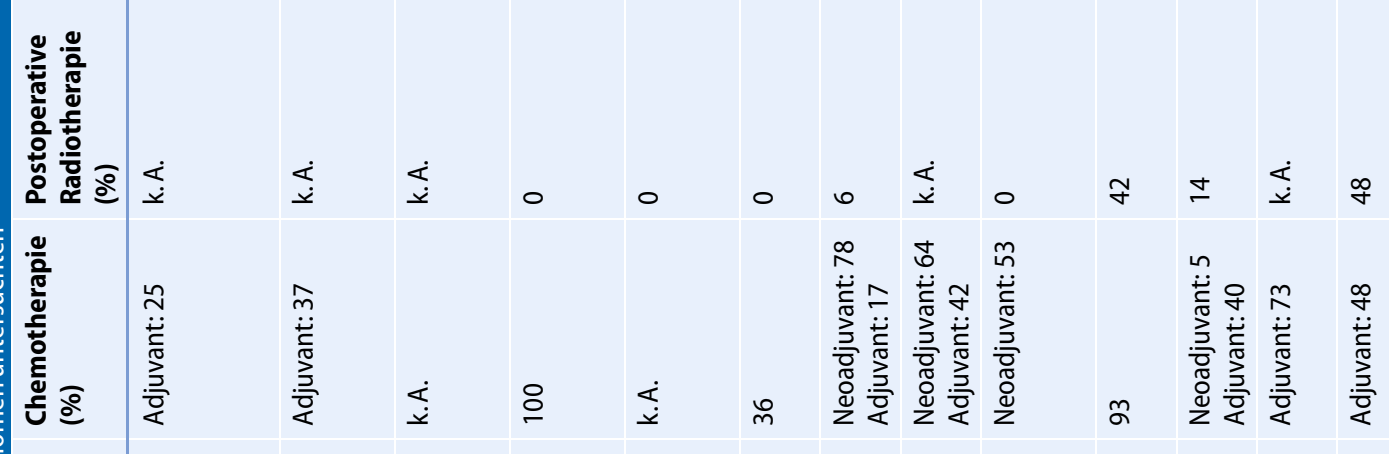

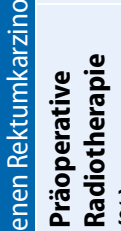

春

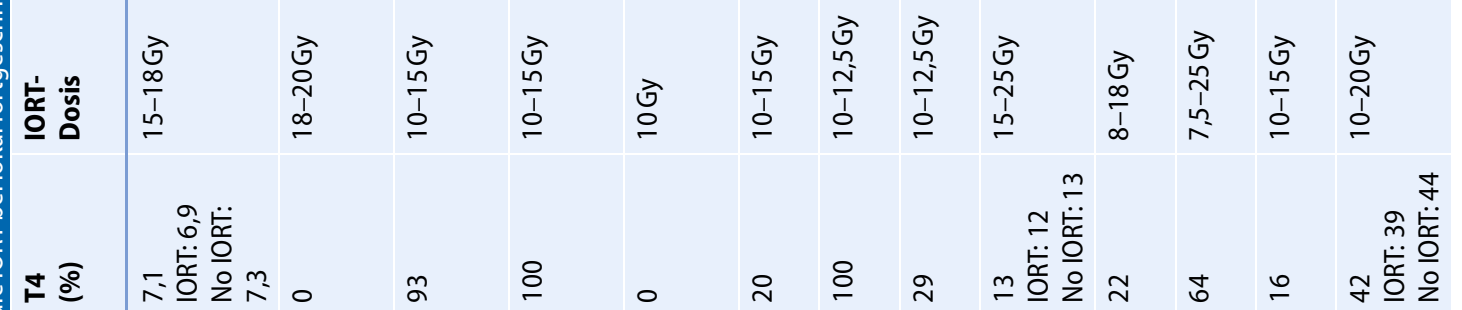

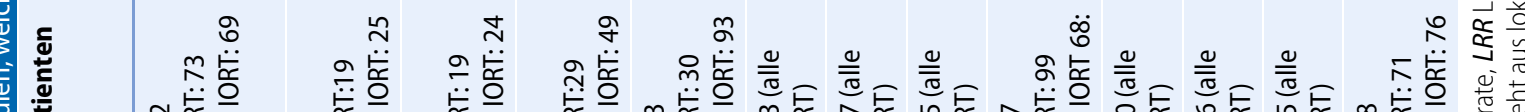

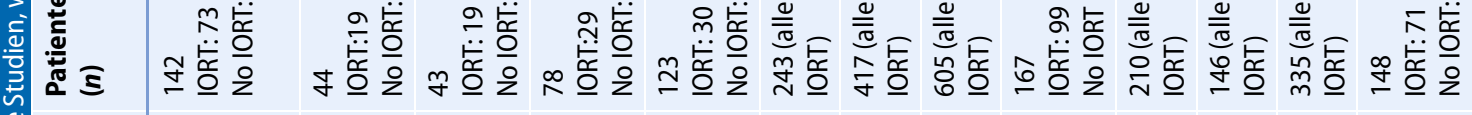

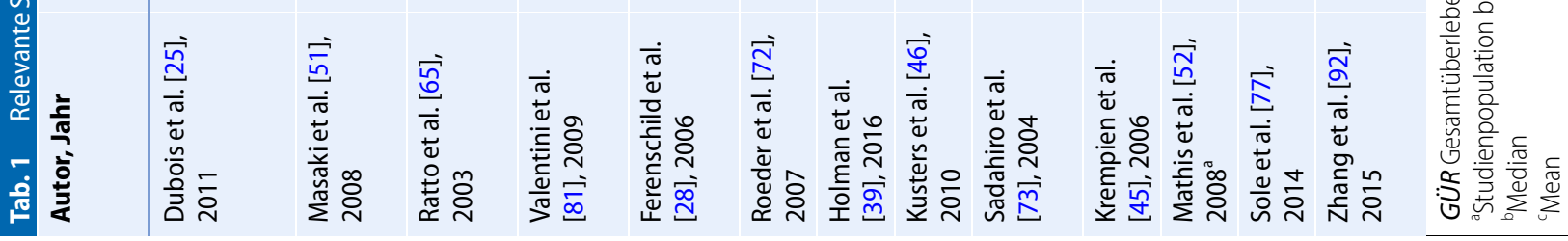


Schweden) durchgeführt, was die Anpassung an verschiedenste Oberflächen erlaubt, sodass auch schwierige Lokalisationen gut therapiert werden können. Die Verschreibung erfolgt typischerweise in einer Gewebstiefe von 0,5 cm [18].

Als dritte Methode der IORT ist die kV-IORT (z. B. Intrabeam, Carl Zeiss AG, Deutschland; Papillon, Ariane Medical Systems Limited, Derby DE1 3BY UK; Axxent Electronic Brachytherapy System, Xoft Inc, Fremont, CA, USA) zu nennen. Mittels dieser lässt sich die Strahlendosis über steile Dosisgradienten besonders nahe an dem Zielvolumen applizieren. Zudem werden hauptsächlich sphärische Applikatoren verwendet, die z. B. in der Therapie von Mammakarzinomen Vorteile bringen [63].

\section{Klinische Anwendung}

\section{Kolorektales Karzinom}

Während die IORT breite klinische Anwendung zur Therapie lokal fortgeschrittener und insbesondere lokal rezidivierender Rektumkarzinome findet und die häufigste Indikation zur IORT in der Viszeralchirurgie darstellt, ist sie im Rahmen eines multidisziplinären Therapiekonzepts bei Kolonkarzinomen nicht etabliert.

Für lokal fortgeschrittene Rektumkarzinome im mittleren und unteren Drittel besteht das gängige Therapiekonzept gemäß Leitlinie aus neoadjuvanter Radiochemotherapie, gefolgt von einer onkologischen Resektion und adjuvanter Chemotherapie. Hierbei nimmt der Resektionsstatus eine zentrale Rolle hinsichtlich lokaler Rezidivraten und Gesamtüberleben ein. In $10 \%$ der Fälle mit tiefer anteriorer Rektumresektion kann jedoch keine R0-Situation erreicht werden [7], sodass besonders diese Patienten von der Durchführung einer IORT profitieren können. Bei primärer Multiviszeralresektion im Becken kann diese Rate noch höher sein.

Die IORT bei primären Rektumkarzinomresektionen wurde bislang nur in zwei randomisiert kontrollierten Studien untersucht $[25,51]$. Beide konnten jedoch - auch aufgrund des Studiendesigns - keinen Vorteil hinsichtlich loka-

Chirurg 2020 -91:743-754 https://doi.org/10.1007/s00104-020-01179-7

(c) Der/die Autor(en) 2020

K. Joechle · E. Gkika · A.-L. Grosu · S. A. Lang · S. Fichtner-Feigl

Intraoperative Strahlentherapie - Indikationen und Optionen in der Viszeralchirurgie

\section{Zusammenfassung}

Hintergrund. Die intraoperative Strahlentherapie (IORT) ermöglicht durch die chirurgische Exposition des Tumors und des Tumorbetts eine hohe Präzision, welche eine hohe Strahlendosis im Bereich des Tumors zulässt und gleichzeitig gesundes Gewebe als den dosislimitierenden Faktor vor Strahlung schützt. Aus diesem Grund bietet die IORT besonders dann einen Vorteil, wenn die lokale Tumorkontrolle das Langzeitüberleben entscheidend beeinflusst und Funktionserhalt ermöglicht.

Ziel der Arbeit. Die in dieser Übersichtsarbeit aufgearbeiteten Erkenntnisse aus der Literaturrecherche erlauben einen evidenzbasierten Umgang hinsichtlich Indikationen und Therapieoptionen der IORT für intraabdominelle Tumoren.

Ergebnisse und Schlussfolgerung. Die

Effektivität der IORT kann anhand der vorhandenen Evidenzlage nicht abschließend beurteilt werden, jedoch ist die IORT als Ergänzung der multimodalen Therapie bei (Rezidiv-)Rektumkarzinomen und Sarkomen aktiv im klinischen Alltag etabliert. Magenund Pankreaskarzinome stellen weitere Indikationen dar; ergänzende Studien sind jedoch notwendig, um die Rolle der IORT hier klar zu definieren. Ein wesentlicher Faktor, damit für Patienten mit primärem Karzinom und insbesondere für Patienten mit lokalem Rezidiv verbesserte lokale Rezidiv- und Überlebensraten erreicht werden können, scheint die Patientenselektion zu sein.

Schlüsselwörter

IORT · Rektumkarzinom · Sarkom ·

Magenkarzinom · Pankreaskarzinom

\section{Intraoperative radiotherapy—Indications and options in visceral surgery}

\section{Abstract}

Background. Intraoperative radiotherapy (IORT) enables a high precision through surgical exposure of the tumor and the tumor bed, which leads to a maximum radiation dose to the tumor while simultaneously protecting normal tissue from radiation as the dose-limiting factor. Therefore, IORT can be particularly advantageous if local tumor control decisively impacts on long-term survival and enables functional preservation. Objective. This review summarizes the knowledge gained from a literature search to enable an evidence-based approach with respect to indications and treatment options of IORT for intra-abdominal tumors.

Results and conclusion. Although the effectiveness of IORT cannot be finally assessed due to limited evidence, IORT is established in the clinical practice as a supplement to the multimodal treatment of (recurrent) rectal cancer and sarcomas. Gastric and pancreatic carcinomas are further indications but additional studies are necessary to clearly define the role of IORT in these tumor entities. An important factor to achieve a benefit with IORT seems to be patient selection in order to obtain good local control of local recurrences as well as overall survival rates for patients with primary or recurrent cancer.

\section{Keywords}

IORT · Rectal carcinoma · Sarcoma · Gastric carcinoma $\cdot$ Pancreatic carcinoma ler Rezidivraten und Gesamtüberleben durch die Ergänzung einer IORT zeigen. Neben einer geringen Studienpopulation $(n=44)$, mit Einschluss von T1und T2-Tumoren in der Studie von Masaki et al. [51], erfolgte in beiden Untersuchungen keine Patientenstratifizierung anhand der MERCURY-Kriterien, sodass die Mehrzahl der eingeschlosse- nen Patienten ein niedriges Risiko für eine R1-Situation und somit für ein Rezidiv hatte. Die fehlende Effektivität der IORT als Ergänzung zur präoperativen, perkutanen Radiotherapie und chirurgischen Resektion werten die Autoren als Folge hoher R0-Resektionsraten und der geringen Anzahl eingeschlossener T4-Tumoren $(n=10 ; 7 \%)$, sodass das lokale 


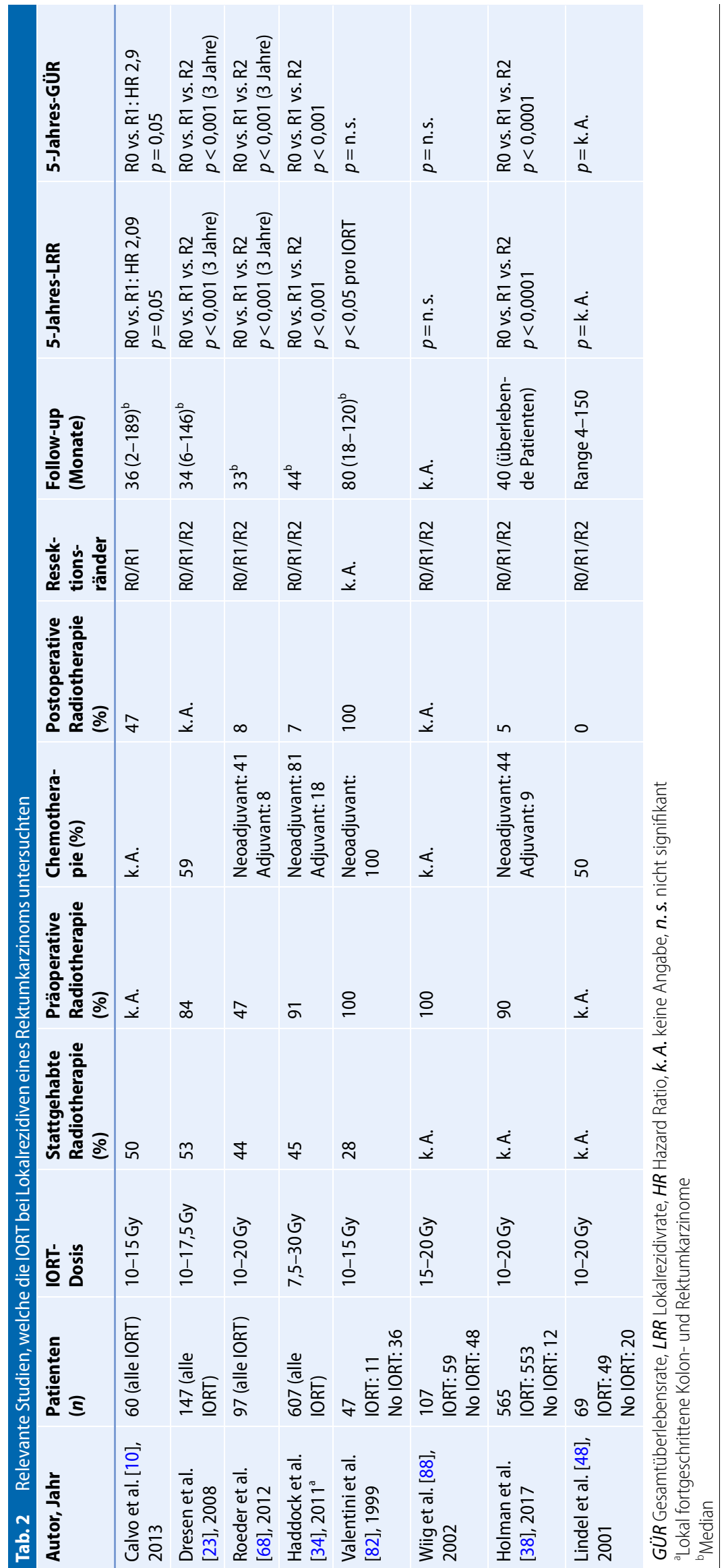

Rezidivrisiko insgesamt als gering eingeschätzt werden müsse. Generell scheint es nach den Prinzipien der totalen mesorektalen Exzision (TME) nicht rational $\mathrm{zu}$ sein, unselektionierte, R0-resezierte primäre Rektumkarzinome durch eine IORT hinsichtlich der Lokalrezidivrate positiv beeinflussen zu können. Vielmehr muss der Fokus auf Hochrisikopatienten gelegt werden.

Analysiert man nichtrandomisierte Studien, welche sich mit der IORT bei lokal fortgeschrittenen Rektumkarzinomen beschäftigen, so ist die Datenlage im Falle kompletter Resektionen ebenfalls nicht eindeutig. Während einige Studien verbesserte Lokalrezidivraten durch IORT auch bei R0-Resektion nachwiesen $[65,81]$, konnten andere Untersuchungen diesen Vorteil nicht zeigen [28]. Eindeutiger zeigt sich die Studienlage im Falle eines positiven Resektionsrandes ( $\mathrm{R}^{+}$-Resektion). Die Durchführung einer IORT verbesserte in dieser Situation nicht nur die Lokalrezidivraten, sondern auch das Gesamtüberleben [28, 39, 46, 72]. Die Ergebnisse selektierter Publikationen sind in $\bullet$ Tab. 1 zusammengefasst $[25,28,39,45,46,51,52,65,72,73,77$, $81,92]$.

Ähnlich wie bei primären, lokal fortgeschrittenen Rektumkarzinomen ist der Resektionsstatus auch bei Lokalrezidiven dieser Tumorentität entscheidend hinsichtlich der Rerezidivraten und des Gesamtüberlebens der Patienten [10, 23, 34, 68]. Im Falle einer R0-Resektion und konsekutiv durchgeführter IORT können 3-Jahres-Lokalrezidivraten von $18 \%$ und ein 3-Jahres-Gesamtüberleben von $80 \%$ erreicht werden. Diese Ergebnisse verschlechtern sich jedoch beim Vorliegen einer R1-Situation mit Lokalrezidivraten von bis zu $59 \%$ und Gesamtüberlebensraten von $37 \%$ [68]. Als Herausforderung stellt sich bei diesen Patienten die im Regelfall vorhandene strahlentherapeutische Vorbehandlung dar, sodass die IORT mittels ihrer Präzision und Minimierung der Strahlenbelastung für gesundes Gewebe eine effektive Möglichkeit darstellt, diese Patienten erneut strahlentherapeutisch zu behandeln. Diesbezüglich konnte der Vorteil der IORT als Ergänzung des multimodalen Therapiekonzeptes durch einige Studien gezeigt werden [82, 88]. 
So betrug das lokalrezidivfreie 5-JahresÜberleben nach R1-Resektion $50 \%$ für Patienten mit IORT vs. $30 \%$ für Patienten ohne IORT [88]. Zudem scheint eine erneute perkutane Bestrahlung der Lokalrezidive die Ergebnisse zu verbessern $[10,38,68]$, insbesondere wenn eine R1-Resektion und geringe Wartezeit zwischen präoperativer perkutaner Bestrahlung und Resektion mit IORT vorliegt [38]. Denn durch Wartezeiten von weniger als 7 Wochen zwischen Beendigung der präoperativen perkutanen Bestrahlung und der IORT könne die Repopulation von Tumorzellen in das Strahlenfeld minimiert werden, womit die Autoren das signifikant reduzierte Lokalrezidivrisiko $(p=0,007)$, besonders für R1-resezierte Patienten, begründen. Die Festlegung der optimalen Zeitspanne zwischen präoperativer und intraoperativer Bestrahlung stellt jedoch eine Herausforderung für Chirurgen und Strahlentherapeuten dar, da in derselben Studie längere Wartezeiten wiederum mit höheren R0-Resektionsraten assoziiert waren [38]. Dies führen die Autoren auf ein nach dieser Zeit erreichtes effektiveres Downstaging des Tumors zurück, sodass eine radikale Resektion erleichtert sei $[38,85]$.

Studien, welche die Durchführung der IORT bei lokalen Rektumkarzinomrezidiven analysierten, sind in $\bullet$ Tab. 2 aufgeführt [10, 23, 34, 38, 48, 68, 82, 88]. Limitierend an diesen Publikationen ist, dass Lokalrezidive je nach Untersuchung unterschiedlich definiert und diagnostiziert wurden und keine Angaben hinsichtlich der Lokalisation der IORT und der daraus resultierenden Effektivität vorhanden sind.

Die Effektivität einer Therapie definiert sich nicht nur aus ihrem Nutzen, sondern auch durch relevante Komplikationen und Risiken. In einer Metaanalyse von Mirnezami et al. [55] konnte gezeigt werden, dass die Ergänzung der multimodalen Therapie des lokal fortgeschrittenen bzw. des lokal rezidivierenden Rektumkarzinoms um die IORT einen Vorteil hinsichtlich der 5-Jahres-Raten lokaler Rezidive, des rezidivfreien Überlebens sowie des Gesamtüberlebens bringt. Zwar treten bei Patienten, welche eine IORT erhalten, mehr Wundkomplikationen auf, dennoch ist die Rate an Gesamtkomplikationen, urologischen Komplikationen und Anastomosenstenosen/-insuffizienzen zwischen den Therapiearmen mit und ohne IORT vergleichbar.

Abschließend lässt sich festhalten, dass die IORT eine effektive Ergänzung der multimodalen Therapie beim Rektumkarzinom darstellt. Patientenselektion ist jedoch ein entscheidender Faktor, sodass bei primären Rektumkarzinomen die Durchführung einer IORT nur bei Patienten mit R1-Resektion und fehlender Möglichkeit der Nachresektion zu empfehlen ist. Auch für Lokalrezidive gilt der Resektionsstatus als stärkster prognostischer Faktor für die Entwicklung eines lokalen Rerezidivs und somit für die Indikation zur IORT.

\section{Sarkome}

Die IORT in der Sarkomtherapie findet sowohl bei Sarkomen der Extremitäten als auch bei intraabdominellen und retroperitonealen Sarkomen Anwendung. Aufgrund der Fokussierung dieses Artikels auf die Indikationen bei intraabdominellen Tumoren wird im Folgenden ausschließlich auf Sarkome in intraabdomineller und retroperitonealer Lokalisation eingegangen.

Aus chirurgisch-technischen und anatomischen Gründen ist bei retroperitonealen Sarkomen trotz radikaler Resektion eine R0-Resektion mit weiten Resektionsrändern manchmal nicht möglich [37, 61, 70]. Dennoch stellt diese einen, wenn nicht den wichtigsten prognostischen Faktor dar [14, 37, 47], sodass mittels IORT als additiver Therapie eine verbesserte lokale Rezidivkontrolle bei knappem Resektionsabstand erreicht werden kann [14, 37, 78].

Die Evidenz der IORT als Teil der multidisziplinären Therapie bei Sarkomen basiert größtenteils auf relativ kleinen retrospektiven Fallserien, welche jedoch hinsichtlich lokaler Rezidivraten und Gesamtüberleben nach IORT vielversprechende Ergebnisse zeigten. Während initiale Studien die IORT häufig kombiniert mit postoperativer, perkutaner Radiatio untersuchten [61], favorisieren aktuellere Studien nach Paradigmenwechsel die Kombination von IORT mit präoperativer, perkutaner Bestrahlung $[32,60,62,79]$. Der Grund hierfür liegt in der präoperativ präziseren Eingrenzung des Strahlenfeldes, was mit reduzierter Toxizität für die umliegenden Organe einhergeht [63]. In einer prospektiven, klinischen Studie [70], in der Patienten mit primären und rezidivierenden Sarkomen $>5 \mathrm{~cm}$ zunächst präoperative perkutane Bestrahlung, gefolgt von Resektion mit IORT erhielten, konnte in einer Zwischenanalyse eine 5-Jahres-Lokalrezidivrate von $28 \%$ erreicht werden.

Relevante Studien sind in $\bullet$ Tab. 3 gelistet $[6,32,40,60-62,67,79,84]$. Häufig sind sowohl Patienten mit primären Sarkomen als auch Patienten mit Sarkomrezidiven eingeschlossen. Obwohl die lokale Kontrolle nach Resektion von Sarkomrezidiven häufig schwieriger gelingt [6], können auch diese Patienten mittels multidisziplinärer Therapie inklusive IORT erfolgreich therapiert werden, wie eine spanische Studie zeigt [12]. $103 \mathrm{~Pa}$ tienten mit lokalem Sarkomrezidiv (Sarkome der Extremitäten eingeschlossen) wiesen 5-Jahres-Lokalrezidivraten, rezidivfreie Überlebensraten und Gesamtüberlebensraten von $27 \%, 43 \%$ und $52 \%$ auf.

Als häufigste Risiken nach IORT bei Sarkomen sind gastrointestinale Toxizität, Ureterstenose und Neuropathie beschrieben. Diese treten in ca. $10-35 \%$ der Fälle auf, mit einem erhöhten Risiko bei zunehmender Strahlendosis. Besonders, um den Funktionserhalt wichtiger Nerven (z.B. Nervus femoralis) zu gewährleisten, wird empfohlen, überlappende Strahlenfelder zu vermeiden und die Strahlendosis auf maximal $12 \mathrm{~Gy} \mathrm{zu}$ beschränken $[54,61,69]$.

\section{Magenkarzinom}

Die Prognose des Magenkarzinoms ist besonders für Patienten mit fortgeschrittenen Tumorstadien, regionalem Lymphknotenbefall oder Peritonealkarzinose eingeschränkt [3], kann jedoch durch perioperative Chemotherapie signifikant verbessert werden. Vielversprechende Ergebnisse zeigt besonders die Durchführung perioperativer Chemotherapie nach dem FLOT(Docetaxel, 
Übersichten

Tab. 3 Relevante Studien, welche die IORT bei retroperitonealen Sarkomen untersuchten

\begin{tabular}{|c|c|c|c|c|c|c|c|c|}
\hline Autor, Jahr & $\begin{array}{l}\text { Patienten } \\
\text { (n) }\end{array}$ & $\begin{array}{l}\text { IORT- } \\
\text { Dosis }\end{array}$ & $\begin{array}{l}\text { Präoperative } \\
\text { Radiotherapie } \\
\text { (\%) }\end{array}$ & $\begin{array}{l}\text { Postoperative } \\
\text { Radiotherapie } \\
\text { (\%) }\end{array}$ & $\begin{array}{l}\text { Resek- } \\
\text { tions- } \\
\text { ränder }\end{array}$ & $\begin{array}{l}\text { Follow-up } \\
\text { (Monate) }\end{array}$ & 5-Jahres-LRR & $\begin{array}{l}\text { 5-Jahres- } \\
\text { GÜR }\end{array}$ \\
\hline $\begin{array}{l}\text { Petersen et al. } \\
\text { [61], } 2002\end{array}$ & $\begin{array}{l}87 \text { (alle IORT) } \\
\text { Rezidive: 50\% }\end{array}$ & $8,75-30 \mathrm{~Gy}$ & 75 & 28 & $\mathrm{R} 0 / \mathrm{R} 1 / \mathrm{R} 2$ & $\begin{array}{l}42 \\
(6-138)^{a}\end{array}$ & $\begin{array}{l}\text { R0 vs. R1 vs. } \\
\text { R2 } \\
p=0,09\end{array}$ & $\begin{array}{l}\text { R0/R1 vs. R2 } \\
p=0,008\end{array}$ \\
\hline $\begin{array}{l}\text { Pierie et al. } \\
\text { [62], } 2006\end{array}$ & $\begin{array}{l}\text { IORT: } 14 \\
\text { No IORT: } 27 \\
\text { Rezidive: } 0 \%\end{array}$ & 10-20 Gy & 100 & 0 & $\mathrm{R} 0 / \mathrm{R} 1 / \mathrm{R} 2$ & $27^{\mathrm{a}}$ & $p=\mathrm{k} . \mathrm{A}$. & $p=0,38$ \\
\hline $\begin{array}{l}\text { Pawlik et al. } \\
{[60], 2006}\end{array}$ & $\begin{array}{l}72 \\
\text { IORT:22 } \\
\text { No IORT: } 50 \\
\text { Rezidive: } 25 \%\end{array}$ & $15 \mathrm{~Gy}$ & 100 & 0 & $\mathrm{R} 0 / \mathrm{R} 1 / \mathrm{R} 2$ & $40^{\mathrm{a}}$ & $p=\mathrm{k} . \mathrm{A}$. & $p=\mathrm{k} . \mathrm{A}$. \\
\hline $\begin{array}{l}\text { Stucky et al. } \\
\text { [79], } 2014\end{array}$ & $\begin{array}{l}63 \\
\text { IORT: } 37 \\
\text { nur Chirurgie: } 26 \\
\text { Rezidive: } 36 \%\end{array}$ & 10-20 Gy & $\begin{array}{l}59 \\
\text { (alle mit IORT) }\end{array}$ & 0 & $\mathrm{R} 0 / \mathrm{R} 1 / \mathrm{R} 2$ & $45^{\mathrm{a}}$ & $\begin{array}{l}p=0,03 \text { pro } \\
\text { IORT }\end{array}$ & $p=$ n.s. \\
\hline $\begin{array}{l}\text { Gronchi et al. } \\
{[32], 2014}\end{array}$ & $\begin{array}{l}83 \\
\text { IORT: } 14 \\
\text { Rezidive: } 24 \%\end{array}$ & $10-12 \mathrm{~Gy}$ & 88 & 0 & $\mathrm{R} 0 / \mathrm{R} 1 / \mathrm{R} 2$ & $\begin{array}{l}58 \\
(36-73)^{a}\end{array}$ & $p=\mathrm{k} . \mathrm{A}$. & $p=\mathrm{k} . \mathrm{A}$. \\
\hline $\begin{array}{l}\text { Ballo et al. [6], } \\
2007\end{array}$ & $\begin{array}{l}83 \\
\text { IORT: } 18 \\
\text { No IORT: } 63 \text { Rezidi- } \\
\text { ve: } 28 \%\end{array}$ & 10-15 Gy & 60 & 40 & $\mathrm{R} 0 / \mathrm{R} 1 / \mathrm{R} 2$ & $47^{\mathrm{a}}$ & $p=0,9$ & $p=\mathrm{k} . \mathrm{A}$. \\
\hline $\begin{array}{l}\text { Roeder et al. } \\
{[67], 2018}\end{array}$ & $\begin{array}{l}156 \text { (alle IORT) } \\
\text { Rezidive: } 56 \%\end{array}$ & $\begin{array}{l}\text { Median } \\
15 \mathrm{~Gy}\end{array}$ & \multicolumn{2}{|c|}{100, nicht spezifiziert } & R0/R1 & $38^{\mathrm{a}}$ & $p=\mathrm{k} . \mathrm{A}$. & $56 \%$ \\
\hline $\begin{array}{l}\text { Wang et al. } \\
\text { [84], } 2017\end{array}$ & $\begin{array}{l}908 \text { (SEER Data- } \\
\text { base) IORT: } 65 \\
\text { No IORT: } 843\end{array}$ & k. A. & \multicolumn{2}{|c|}{ 96, nicht spezifiziert } & k. A. & k. A. & $p=\mathrm{k} . \mathrm{A}$. & $p=0,31$ \\
\hline $\begin{array}{l}\text { Hull et al. [40], } \\
2017\end{array}$ & $\begin{array}{l}46 \\
\text { IORT: } 16 \\
\text { No IORT: } 30 \\
\text { Rezidive: } 15 \%\end{array}$ & $\begin{array}{l}\text { Median } \\
10 \mathrm{~Gy}\end{array}$ & 100 & 15 & R0/R1 & $\begin{array}{l}53(\mathrm{IQR} \\
30-77)\end{array}$ & $p=\mathrm{k} . \mathrm{A}$. & $81 \%$ \\
\hline
\end{tabular}

Oxaliplatin, Leucovorin und Fluoruracil)-Schema [3,4], die auch den aktuellen Standard ergänzend zur Resektion eines Magenkarzinoms darstellt. Eine Therapiestrategie, welche besonders in den 1990er-Jahren untersucht und angewendet wurde, ist die IORT, mit dem Ziel lokale Rezidivraten zu minimieren. Nach Studienlage profitierten hierbei besonders Patienten mit Magenkarzinom im Stadium II/III und Lymphknotenbefall $[24,64,76,91]$, wie relevante Publikationen in - Tab. 4 zeigen [13, 24, 31, $64,76,91]$. Eine Metaanalyse konnte die verbesserten Lokalrezidivraten durch die Ergänzung der multimodalen Therapie um die IORT beim Magenkarzinom validieren, zeigte jedoch auch, dass die IORT nur bei Patienten mit einem Magenkarzinom im Stadium III einen Einfluss auf das Überleben hatte [90]. Zudem waren diese Ergebnisse mit dem Grad der durchgeführten Lymphadenektomie vergesellschaftet, sodass hauptsächlich Patienten, welche die IORT kombiniert mit limitierter Lymphadenektomie erhielten, verbesserte Überlebensraten zeigten [17, 64]. Diesbezüglich muss ausdrücklich erwähnt werden, dass die Durchführung einer IORT keine schlecht oder nur begrenzt durchgeführte chirurgische Resektion ersetzt. Auch das Komplikationsprofil nach IORT ist nicht eindeutig; während einige Studien ähnliche Komplikationsraten nach IORT verglichen zur alleinigen Resektion beschrieben [17, 29, 76], zeigten andere erhöhte Morbiditätsraten und Spätkomplikationen nach IORT [24, 91].

Obwohl in den aufgeführten Studien gute Ergebnisse hinsichtlich lokaler Rezidivkontrolle durch die IORT erzielt wur- den, resultierte dies nicht in einem Überlebensvorteil für alle Patientengruppen. Dies lässt sich womöglich auf das Auftreten von Fernmetastasen zurückführen, sodass systemisch effektivere Therapiestrategien zur Behandlung des Magenkarzinoms notwendig sind und damit die IORT aktuell nicht zur Standardtherapie des Magenkarzinoms gehört.

\section{Pankreaskarzinom}

Trotz kurativer Resektion haben Patienten mit Pankreaskarzinom eine sehr eingeschränkte Prognose. Aktuell konnten verbesserte Resektions- und Überlebensraten durch neoadjuvante Therapieregime mit FOLFIRINOX oder Nab-Paclitaxel ( \pm Radiatio) erreicht werden [16, $33,56]$. Da häufig trotz intensiver Vorbehandlung keine R0-Resektion bei lo- 


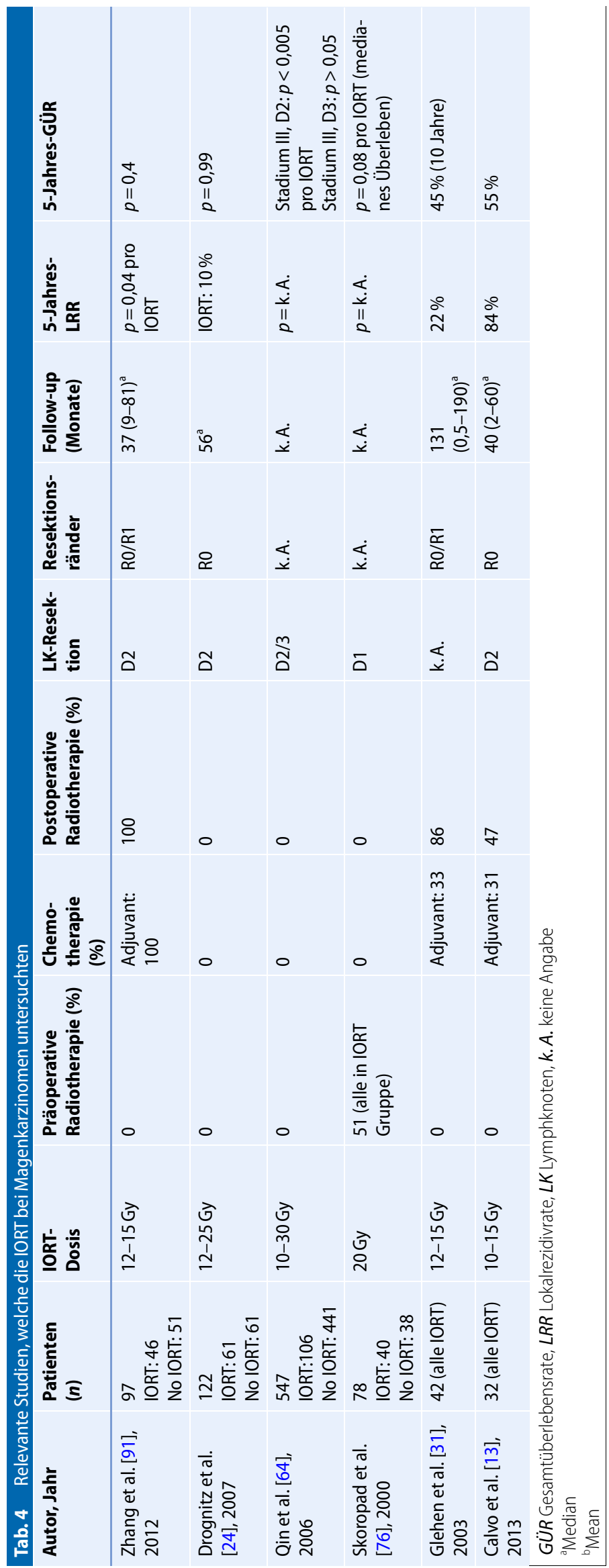

kal fortgeschrittenen oder Borderline-resektablen Pankreaskarzinomen möglich ist, kann die IORT eine gute, additive Therapie darstellen, um trotzdem geringe Lokalrezidivraten zu erreichen. Diese vielversprechenden, neoadjuvanten Therapiestrategien in Verbindung mit der IORT wurden bisher nur durch eine Studie von Keane et al. [43] untersucht. Hierbei wurden 68 Patienten mit Borderlineresektablem oder lokal fortgeschrittenem Pankreaskarzinom eingeschlossen, wovon 41 nach neoadjuvanter Vorbehandlung resektabel waren. Davon wiederum erhielten 22 Patienten aufgrund knapper oder positiver Resektionsränder an diesen und im Tumorbett eine IORT und zeigten mit 35,1 und 21 Monaten ein verbessertes medianes Gesamt- bzw. progressionsfreies Überleben im Vergleich zu Patienten ohne IORT mit einem medianen Gesamt- bzw. progressionsfreiem Überleben von 24,5 und 16,3 Monaten. Jedoch erwies sich die IORT auch bei denjenigen Patienten, die irresektabel waren und nur eine IORT des Tumors sowie eine Gastrojejunostomie erhielten, mit einem medianen Gesamtüberleben von 24,8 Monaten und progressionsfreiem Überleben von 16,1 Monaten als effektiv. Diese beeindruckenden Daten trotz in situ belassenen Tumors bedürfen laut den Autoren weiterer Studien, da aufgrund des Paradigmenwechsels keine vergleichbaren Daten vorlägen. Im Gegensatz dazu stellten ältere Studien, welche die IORT bei lokal fortgeschrittenen und nichtresektablen Pankreaskarzinomen untersuchten, keinen Überlebensvorteil bei signifikant verbesserten Lokalrezidivraten fest $[8,15,41,50]$. Diese sind in 0 Tab. 5 zusammengefasst [ 8 , $15,41,43,50]$.

Im Gegensatz zu den lokal irresektablen oder Borderline-resektablen Pankreaskarzinomen konnten für primär resektable Pankreaskarzinome nicht nur verminderte Lokalrezidivraten $[5,11,75$, 80], sondern durch einige Studien auch verbesserte Gesamtüberlebensraten bestätigt werden $[5,66,80]$. Jedoch wurde in nur einer dieser Studien bei 39\% der Patienten eine adjuvante Chemotherapie durchgeführt, diese hatte in der multivariaten Analyse keinen Einfluss auf das Gesamtüberleben [66]. Es ließen sich ne- 


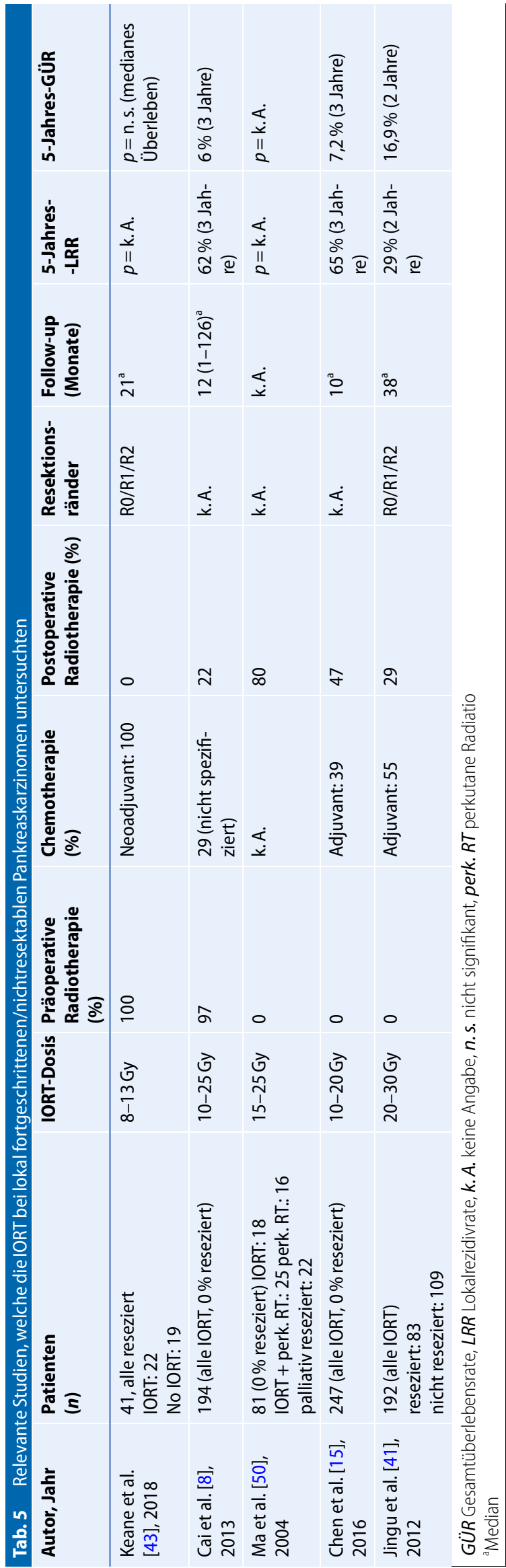

ben der IORT das Tumorstadium [66], eine R0-Resektion [11] und die Durchführung einer neoadjuvanten Therapie [80] als wichtige prognostische Faktoren hinsichtlich des Gesamtüberlebens nachweisen. Relevante Studien sind in $\mathbf{0}$ Tab. 6 aufgeführt $[5,11,53,57,66,75,80]$.

Eine bisher nicht gut untersuchte Indikation zur IORT beim Pankreaskarzinom stellt das isolierte Lokalrezidiv dar. In einer diesbezüglichen Studie [71] wurden 36 Patienten mit Lokalrezidiv mittels Resektion, IORT und adjuvanter Radiatio $(86 \%)$ behandelt. Dabei konnten mit einer 2-Jahres-Lokalrezidivrate von 33\% und 2-Jahres-Gesamtüberlebensrate von $45 \%$ vielversprechende Ergebnisse verzeichnet werden.

Die vorgestellten Studien sind trotz guter Ergebnisse und geringem Toxizitätsprofil der durchgeführten IORT hauptsächlich durch ihr größtenteils retrospektives Design sowie unterschiedliche Einschlusskriterien ( $\mathrm{R}^{+}$-Resektionen, neo-/adjuvante Therapieregimen) limitiert. Deswegen wird von einigen Autoren eine Phase-III-Studie gefordert, um die Rolle der IORT zur Behandlung des Pankreaskarzinoms, insbesondere in Kombination mit neuen neoadjuvanten Therapiestrategien, genauer $\mathrm{zu}$ definieren [44].

\section{Ausblick}

Der Hauptmechanismus, welcher lange Zeit das Ansprechen solider Tumoren auf die Strahlentherapie erklärte, ist die irreparable Dann-Schädigung, welche wiederum durch den Prozess der Apoptose, Seneszenz oder Autophagie zum direkten Zelltod führt [26, 74]. Neben immunsuppressiver Effekte der Strahlentherapie wie z. B. der Induktion einer Lymphozytopenie [49] wurde in den vergangenen Jahren zunehmend auch eine immunstimulierende Komponente der Strahlentherapie untersucht und beschrieben. So werden durch den mittels Radiotherapie induzierten, immunogenen Zelltod inflammatorische Zytokine ausgeschüttet und Antigene freigesetzt, welche den T-Zellen durch dendritische Zellen zur Aktivierung der zytotoxischen Immunantwort cross-präsentiert werden [42]. Gleichzeitig wird 


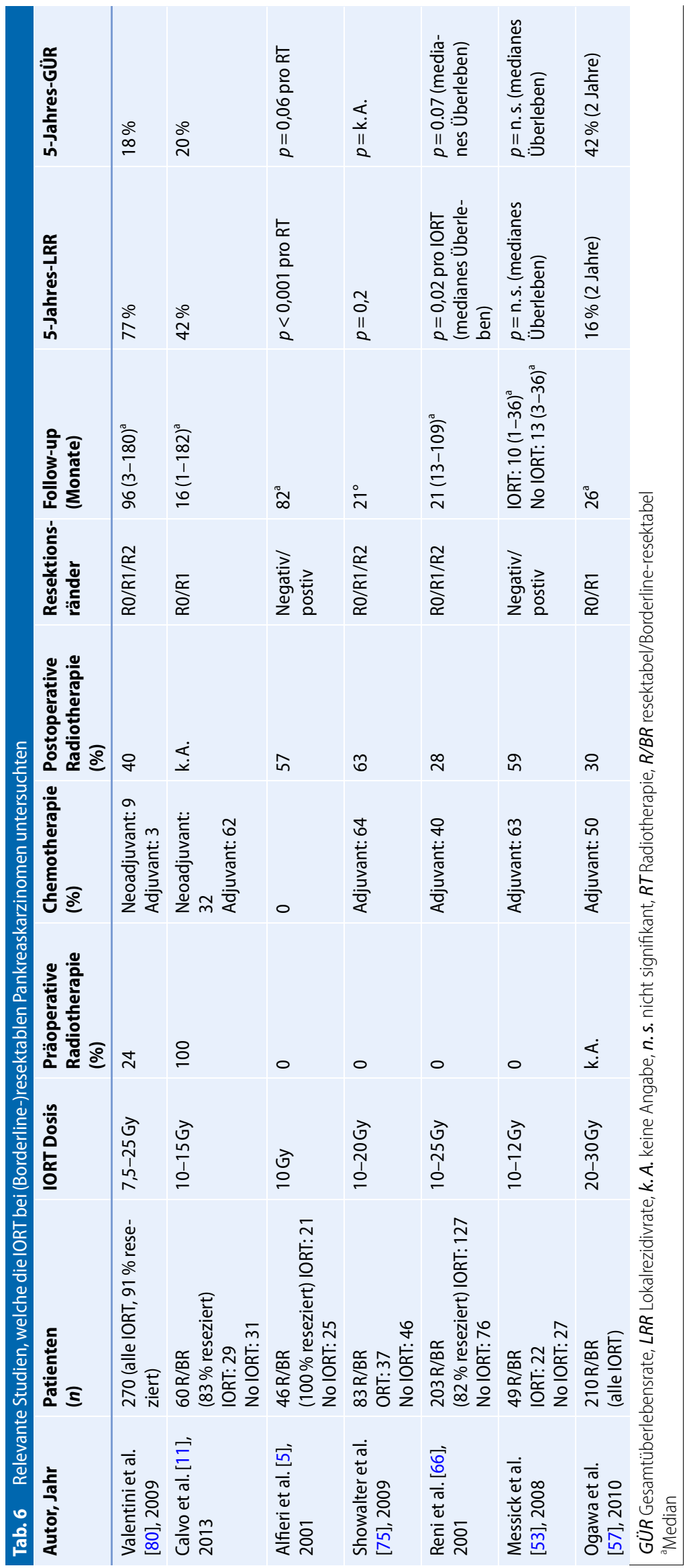

über die Freisetzung von Chemokinen eine vermehrte Infiltration zytotoxischer T-Zellen in den Tumor erreicht, die im Anschluss die Tumorzellen z.B. durch Hochregulation von MHC-1 erkennen und abtöten können. Dies führt nicht nur zur Unterdrückung des strahlentherapeutisch behandelten Tumors, sondern auch vorhandener Metastasen, was als abscopaler Effekt bezeichnet wird [19].

Da die Antitumorimmunität, welche durch Strahlentherapie allein erreicht wird, gewöhnlich hinsichtlich des abscopalen Effekts limitiert ist, kann diese durch die Kombination von Radiatio und Immuncheckpoint-Inhibitoren (AntiCTLA-4- und/oder Anti-PD-1- oder PD-L1-monoklonale Antikörper) gesteigert werden [19]. Denn diese ermöglichen mittels Blockade von CTLA-4 eine verstärkte die T-Zell Aktivierung [89] bzw. über die Inhibition der PD-1/PDL1-Achse eine gesteigerte T-Zell-Effektorfunktion [58]. Diese synergistischen Effekte konnten in präklinischen Studien mittels unterschiedlicher In-vivoModelle verifiziert werden [20-22]. Da sich mit höherer Strahlendosis auch eine erhöhte Immunmodulation nachweisen lässt [86, 87], könnte die Kombination der IORT mit Immuncheckpoint-Inhibitoren eine weitere vielversprechende Anwendung der IORT darstellen. Diese Rationale wurde in einem kürzlich veröffentlichten Review im Rahmen der Behandlung von Hirnmetastasen sehr gut dargestellt [36], muss jedoch durch weitere Studien noch genauer untersucht werden. Denn bisher ist aufgrund der aufgeführten Studien die Effektivität der IORT nicht gänzlich geklärt, sodass die Kombination dieser mit CheckpointInhibitoren ein zukünftiges, aber bisher rein potenzielles Anwendungsgebiet darstellt.

\section{Fazit für die Praxis}

Die IORT dient der Ergänzung der multimodalen Therapiestrategie bei intraabdominellen und retroperitonealen Karzinomen und ist bei Rektumkarzinomen und Sarkomen aktiv im klinischen Alltag etabliert. Die Effektivität der IORT ist jedoch anhand der vorhandenen und aufgeführten Evidenzlage nicht 


\section{abschließend zu beurteilen. Ein häufig diskutierter Hinweis hinsichtlich der Wirksamkeit der IORT ist die erreichbare Dosiseskalation bei geringem Kom- plikationsprofil. Wichtig dabei scheint besonders die Patientenselektion, so- dass mittels IORT nicht nur Patienten mit primärem Karzinom, sondern ins- besondere bei Patienten mit lokalem Rezidiv verbesserte lokale Rezidiv- und Überlebensraten erreichen können.}

\section{Korrespondenzadresse}

\section{Stefan Fichtner-Feigl}

Klinik für Allgemein- und Viszeralchirurgie, Universitätsklinikum Freiburg

Hugstetterstraße 55, 79106 Freiburg,

Deutschland

stefan.fichtner@uniklinik-freiburg.de

Funding. Open Access funding provided by Projekt DEAL.

\section{Einhaltung ethischer Richtlinien}

Interessenkonflikt. K. Joechle, E. Gkika, A.-L. Grosu, S.A. Lang und S. Fichtner-Feigl geben an, dass kein Interessenkonflikt besteht.

Für diesen Beitrag wurden von den Autoren keine Studien an Menschen oder Tieren durchgeführt. Für die aufgeführten Studien gelten die jeweils dort angegebenen ethischen Richtlinien.

Open Access. Dieser Artikel wird unter der Creative Commons Namensnennung 4.0 International Lizenz veröffentlicht, welche die Nutzung, Vervielfältigung, Bearbeitung, Verbreitung und Wiedergabe in jeglichem Medium und Format erlaubt, sofern Sie den/die ursprünglichen Autor(en) und die Quelle ordnungsgemäß nennen, einen Link zur Creative Commons Lizenz beifügen und angeben, ob Änderungen vorgenommen wurden.

Die in diesem Artikel enthaltenen Bilder und sonstiges Drittmaterial unterliegen ebenfalls der genannten Creative Commons Lizenz, sofern sich aus der Abbildungslegende nichts anderes ergibt. Sofern das betreffende Material nicht unter der genannten Creative Commons Lizenz steht und die betreffende Handlung nicht nach gesetzlichen Vorschriften erlaubt ist, ist für die oben aufgeführten Weiterverwendungen des Materials die Einwilligung des jeweiligen Rechteinhabers einzuholen.

Weitere Details zur Lizenz entnehmen Sie bitte der Lizenzinformation auf http://creativecommons.org/ licenses/by/4.0/deed.de.

\section{Literatur}

1. Abe M, Yamano K, Imura T et al (1969) Intraoperative radiotherapy of abdominal tumors. 1.
Intraoperative irradiation of carcinoma of the pancreas head and biliary system. Nippon Igaku Hoshasen Gakkai Zasshi 29:75-85

2. Abe M, Yamano K, Matsuda S (1970) Intraoperative radiotherapy of abdominal tumors. 3 . Intraoperative irradiation to carcinoma of the colon and retroperitoneal tumors. Nihon Igaku Hoshasen Gakkai Zasshi 29:1488-1493

3. Al-Batran SE, Hofheinz RD, Pauligk C et al (2016) Histopathological regression after neoadjuvant docetaxel, oxaliplatin, fluorouracil, and leucovorin versus epirubicin, cisplatin, and fluorouracil or capecitabine in patients with resectable gastric or gastro-oesophageal junction adenocarcinoma (FLOT4-AIO): results from the phase 2 part of a multicentre, open-label, randomised phase $2 / 3$ trial. Lancet Oncol 17:1697-1708

4. Al-Batran SE, Homann N, Pauligk C et al (2019) Perioperative chemotherapy with fluorouracil plus leucovorin, oxaliplatin, and docetaxel versus fluorouracil or capecitabine plus cisplatin and epirubicin for locally advanced, resectable gastric or gastro-oesophageal junction adenocarcinoma (FLOT4): a randomised, phase $2 / 3$ trial. Lancet 393(10184):1948-1957

5. Alfieri S, Morganti AG, Di Giorgio A et al (2001) Improved survival and local control after intraoperative radiation therapy and postoperative radiotherapy: a multivariate analysis of 46 patients undergoing surgery for pancreatic head cancer. Arch Surg 136:343-347

6. Ballo MT, Zagars GK, Pollock RE et al (2007) Retroperitoneal soft tissue sarcoma: an analysis of radiation and surgical treatment. Int J Radiat Oncol Biol Phys 67:158-163

7. Bonjer HJ, Deijen CL, Abis GA etal (2015) A randomized trial of laparoscopic versus open surgery for rectal cancer. N Engl J Med 372:1324-1332

8. Cai S, Hong TS, Goldberg SI et al (2013) Updated long-term outcomes and prognostic factors for patients with unresectable locally advanced pancreatic cancer treated with intraoperative radiotherapy at the Massachusetts General Hospital, 1978 to 2010. Cancer 119:4196-4204

9. Calvo F, Sole C, Herranz Retal (2013) Intraoperative radiotherapy with electrons: fundamentals, results, and innovation. ecancermedicalscience 7:339

10. Calvo FA, Sole CV, Alvarez De Sierra P et al (2013) Prognostic impact of external beam radiation therapy in patients treated with and without extended surgery and intraoperative electrons for locally recurrent rectal cancer: 16-year experience in a single institution. Int J Radiat Oncol Biol Phys 86:892-900

11. Calvo FA, Sole CV, Atahualpa F et al (2013) Chemoradiation for resected pancreatic adenocarcinoma with or without intraoperative radiation therapy boost: long-term outcomes. Pancreatology 13:576-582

12. Calvo FA, Sole CV, Cambeiro M et al (2014) Prognostic value of external beam radiation therapy in patients treated with surgical resection and intraoperative electron beam radiation therapy for locally recurrent soft tissue sarcoma: a multicentric long-term outcome analysis. Int J Radiat Oncol Biol Phys 88:143-150

13. Calvo FA, Sole CV, Obregon R et al (2013) Intraoperative radiotherapy for the treatment of resectable locally advanced gastric adenocarcinoma: topography of locoregional recurrences and long-term outcomes. Clin Transl Oncol 15:443-449

14. Catton CN, O'Sullivan B, Kotwall C et al (1994) Outcome and prognosis in retroperitoneal soft tissue sarcoma. Int J Radiat Oncol Biol Phys 29:1005-1010

15. Chen Y, Che X, Zhang J et al (2016) Long-term results of intraoperative electron beam radiation therapy for nonmetastatic locally advanced pancreatic cancer: retrospective cohort study, 7-year experience with 247 patients at the National Cancer Center in China. Medicine 95:e4861

16. Conroy T, Bachet JB, Ayav A et al (2016) Current standards and new innovative approaches for treatment of pancreatic cancer. Eur J Cancer 57:10-22

17. Coquard R, Ayzac L, Gilly FN et al (1997) Intraoperative radiation therapy combined with limited lymph node resection in gastric cancer: an alternative to extended dissection? Int J Radiat Oncol Biol Phys 39:1093-1098

18. Debenham BJ, Hu KS, Harrison LB (2013) Present status and future directions of intraoperative radiotherapy. Lancet Oncol 14:e457-e464

19. Demaria S, Golden EB, Formenti SC (2015) Role of local radiation therapy in cancer immunotherapy. JAMA Oncol 1:1325-1332

20. Deng L, Liang $H$, Burnette B et al (2014) Irradiation and anti-PD-L1 treatment synergistically promote antitumor immunity in mice. J Clin Invest 124:687-695

21. Dewan MZ, Galloway AE, Kawashima N et al (2009) Fractionated but not single-dose radiotherapy induces an immune-mediated abscopal effect when combined with anti-CTLA-4 antibody. Clin Cancer Res 15:5379-5388

22. DovediSJ, Adlard AL, Lipowska-Bhalla Getal (2014) Acquired resistance to fractionated radiotherapy can be overcome by concurrent PD-L1 blockade. Cancer Res 74:5458-5468

23. Dresen RC, Gosens MJ, Martijn Hetal (2008) Radical resection after IORT-containing multimodality treatment is the most important determinant for outcome in patients treated for locally recurrent rectal cancer. Ann Surg Oncol 15:1937-1947

24. Drognitz O, Henne K, Weissenberger C et al (2008) Long-term results after intraoperative radiation therapy for gastric cancer. Int J Radiat Oncol Biol Phys 70:715-721

25. Dubois JB, Bussieres E, Richaud P et al (2011) Intraoperative radiotherapy of rectal cancer: results of the French multi-institutional randomized study. Radiother Oncol 98:298-303

26. Eriksson D, Stigbrand T (2010) Radiation-induced cell death mechanisms. Tumour Biol 31:363-372

27. Esposito A, Sakellaris T, Limede Pet al (2016) Effects of shielding on pelvic and abdominal IORT dose distributions. Phys Med 32:1397-1404

28. Ferenschild FT, Vermaas M, Nuyttens JJ et al (2006) Value of intraoperative radiotherapy in locally advanced rectal cancer. Dis Colon Rectum 49:1257-1265

29. Fu S, Lu JJ, Zhang Q et al (2008) Intraoperative radiotherapy combined with adjuvant chemoradiotherapy for locally advanced gastric adenocarcinoma. Int J Radiat Oncol Biol Phys 72:1488-1494

30. Fukuda M, Abe M, Yamano K et al (1969) Intraoperative radiotherapy of abdominal tumors. 2. Intraoperative irradiation to carcinoma of the stomach. Nihon Igaku Hoshasen Gakkai Zasshi 29:390-399

31. Glehen O, Peyrat P, Beaujard AC et al (2003) Pattern of failures in gastric cancer patients with lymph node involvement treated by surgery, intraoperative and external beam radiotherapy. Radiother Oncol 67:171-175 
32. Gronchi A, De Paoli A, Dani C et al (2014) Preoperative chemo-radiation therapy for localised retroperitoneal sarcoma: a phase I-II study from the Italian Sarcoma Group. Eur J Cancer 50:784-792

33. Hackert T, Sachsenmaier M, Hinz U et al (2016) Locally advanced pancreatic cancer: neoadjuvant therapy with folfirinox results in resectability in $60 \%$ of the patients. Ann Surg 264:457-463

34. Haddock MG, Miller RC, Nelson Het al (2011) Combined modality therapy including intraoperative electron irradiation for locally recurrent colorectal cancer. Int J Radiat Oncol Biol Phys 79:143-150

35. Hensley FW (2017) Present state and issues in IORT physics. Radiat Oncol 12:37

36. Herskind C, Wenz F, Giordano FA (2017) Immunotherapy combined with large fractions of radiotherapy: stereotactic radiosurgery for brain metastases-implications for intraoperative radiotherapy after resection. Front Oncol 7:147

37. Heslin MJ, Lewis JJ, Nadler E et al (1997) Prognostic factors associated with long-term survival for retroperitoneal sarcoma: implications for management. JClin Oncol 15:2832-2839

38. Holman FA, Bosman SJ, Haddock MG et al (2017) Results of a pooled analysis of IOERT containing multimodality treatment for locally recurrent rectal cancer: results of 565 patients of two major treatment centres. Eur J Surg Oncol 43:107-117

39. Holman FA, Haddock MG, Gunderson LL et al (2016) Results of intraoperative electron beam radiotherapy containing multimodality treatment for locally unresectable T4 rectal cancer: a pooled analysis of the Mayo Clinic Rochester and Catharina Hospital Eindhoven.J Jastrointest Oncol 7:903-916

40. Hull MA, Molina G, Niemierko A et al (2017) Improved local control with an aggressive strategy of preoperative (with or without intraoperative) radiation therapy combined with radical surgical resection for retroperitoneal sarcoma. J Surg Onco 115:746-751

41. Jingu $K$, Tanabe $T$, Nemoto $K$ et al (2012) Intraoperative radiotherapy for pancreatic cancer: 30-year experience in a single institution in Japan. Int JRadiat Oncol Biol Phys 83:e507-e511

42. Joyce JA, Fearon DT (2015) T cell exclusion, immune privilege, and the tumor microenvironment. Science 348:74-80

43. Keane FK, Wo JY, Ferrone CR et al (2018) Intraoperative radiotherapy in the era of intensive neoadjuvant chemotherapy and chemoradiotherapy for pancreatic adenocarcinoma. Am J Clin Oncol 41:607-612

44. Krempien R, Roeder F (2017) Intraoperative radiation therapy (IORT) in pancreatic cancer. Radiat Oncol 12:8

45. Krempien R, Roeder F, Oertel S et al (2006) Longterm results of intraoperative presacral electron boost radiotherapy (IOERT) in combination with total mesorectal excision (TME) and chemoradiation in patients with locally advanced rectal cancer. Int JRadiat Oncol Biol Phys 66:1143-1151

46. Kusters M, Valentini V, Calvo FA et al (2010) Results of European pooled analysis of IORT-containing multimodality treatment for locally advanced rectal cancer: adjuvant chemotherapy prevents local recurrence rather than distant metastases. Ann Oncol 21:1279-1284

47. Lewis JJ, Leung D, Woodruff JM et al (1998) Retroperitoneal soft-tissue sarcoma: analysis of 500 patients treated and followed at a single institution. Ann Surg 228:355-365

48. Lindel K, Willett CG, Shellito PC et al (2001) Intraoperative radiation therapy for locally advanced recurrent rectal or rectosigmoid cancer. Radiother Oncol 58:83-87

49. Liu Y, Dong Y, Kong L et al (2018) Abscopal effect of radiotherapy combined with immune checkpoint inhibitors. JHematol Oncol 11:104

50. Ma HB, Di ZL, Wang XJ et al (2004) Effect of intraoperative radiotherapy combined with external beam radiotherapy following internal drainage for advanced pancreatic carcinoma. World J Gastroenterol 10:1669-1771

51. Masaki T, Takayama M, Matsuoka H et al (2008) Intraoperative radiotherapy for oncological and function-preserving surgery in patients with advanced lower rectal cancer. Langenbecks Arch Surg 393:173-180

52. Mathis KL, Nelson H, Pemberton JH et al (2008) Unresectable colorectal cancer can be cured with multimodality therapy. Ann Surg 248:592-598

53. MessickC, Hardacre JM, McGee MF etal (2008) Early experience with intraoperative radiotherapy in patients with resected pancreatic adenocarcinoma. Am J Surg 195:308-311 (discussion 312)

54. Miller RC, Haddock MG, Petersen IA et al (2006) Intraoperative electron-beam radiotherapy and ureteral obstruction. Int J Radiat Oncol Biol Phys 64:792-798

55. Mirnezami R, Chang GJ, Das P et al (2013) Intraoperative radiotherapy in colorectal cancer: systematic review and meta-analysis of techniques, longterm outcomes, and complications. Surg Oncol 22:22-35

56. Murphy JE, Wo JY, Ryan DP et al (2018) Total neoadjuvant therapywith FOLFIRINOXfollowed by individualized chemoradiotherapy for borderline resectable pancreatic adenocarcinoma: a phase 2 clinical trial. JAMA Oncol 4:963-969

57. Ogawa K, Karasawa K, Ito Y et al (2010) Intraoperative radiotherapy for resected pancreatic cancer: a multi-institutional retrospective analysis of 210 patients. Int J Radiat Oncol Biol Phys 77:734-742

58. Pardoll DM (2012) The blockade of immune checkpoints in cancer immunotherapy. Nat Rev Cancer 12:252-264

59. Paunesku T, Woloschak GE (2017) Future directions of intraoperative radiation therapy: a brief review. Front Oncol 7:300

60. Pawlik TM, Pisters PW, Mikula L et al (2006) Longterm results of two prospective trials of preoperative external beam radiotherapy for localized intermediate- or high-grade retroperitoneal soft tissue sarcoma. Ann Surg Oncol 13:508-517

61. Petersen IA, Haddock MG, Donohue JH et al (2002) Use of intraoperative electron beam radiotherapy in the management of retroperitoneal soft tissue sarcomas. Int JRadiat Oncol Biol Phys 52:469-475

62. Pierie JP, Betensky RA, Choudry U et al (2006) Outcomes in a series of 103 retroperitoneal sarcomas. Eur J Surg Oncol 32:1235-1241

63. Pilar A, Gupta M, Ghosh Laskar S et al (2017) Intraoperative radiotherapy: review of techniques and results. Ecancermedicalscience 11:750

64. Qin HL, Lin CH, Zhang XL (2006) Evaluation of intraoperative radiotherapy for gastric carcinoma with D2 and D3 surgical resection. World J Gastroenterol 12:7033-7037

65. Ratto C, Valentini V, Morganti AG et al (2003) Combined-modality therapy in locally advanced primary rectal cancer. Dis Colon Rectum 46:59-67

66. Reni M, Panucci MG, Ferreri AJ et al (2001) Effect on local control and survival of electron beam intraoperative irradiation for resectable pancreatic adenocarcinoma. Int J Radiat Oncol Biol Phys 50:651-658
67. Roeder F, Alldinger I, Uhl Met al (2018) Intraoperative electron radiation therapy in retroperitoneal sarcoma. Int J Radiat Oncol Biol Phys 100:516-527

68. Roeder F, Goetz JM, Habl G et al (2012) Intraoperative electron radiation therapy (IOERT) in the management of locally recurrent rectal cancer. BMC Cancer 12:592

69. Roeder F, Krempien R (2017) Intraoperative radiation therapy (IORT) in soft-tissue sarcoma. Radiat Oncol 12:20

70. Roeder F, Schulz-Ertner D, Nikoghosyan AV et al (2012) A clinical phase I/II trial to investigate preoperative dose-escalated intensity-modulated radiation therapy (IMRT) and intraoperative radiation therapy (IORT) in patients with retroperitonea soft tissue sarcoma. BMC Cancer 12:287

71. Roeder F, Timke C, Uhl M et al (2012) Aggressive local treatment containing intraoperative radiation therapy (IORT) for patients with isolated local recurrences of pancreatic cancer: a retrospective analysis. BMC Cancer 12:295

72. Roeder F, Treiber M, Oertel S et al (2007) Patterns of failure and local control after intraoperative electron boost radiotherapy to the presacral space in combination with total mesorectal excision in patients with locally advanced rectal cancer. Int J Radiat Oncol Biol Phys 67:1381-1388

73. Sadahiro S, Suzuki T, Ishikawa Ketal (2004) Preoperative radio/chemo-radiotherapy in combination with intraoperative radiotherapy for T3-4Nx rectal cancer. Eur J Surg Oncol 30:750-758

74. Serre R, Benzekry S, Padovani L et al (2016) Mathematical modeling of cancerimmunotherapy and its synergy with radiotherapy. Cancer Res 76:4931-4940

75. Showalter TN, Rao AS, Anne PR et al (2009) Does intraoperative radiation therapy improve local tumor control in patients undergoing pancreaticoduodenectomy for pancreatic adenocarcinoma? A propensity score analysis. Ann Surg Oncol 16:2116-2122

76. Skoropad VY, Berdov BA, Mardynski YS et al (2000) A prospective, randomized trial of pre-operative and intraoperative radiotherapy versus surgery alone in resectable gastric cancer. Eur J Surg Onco 26:773-779

77. Sole CV, Calvo FA, Serrano J et al (2014) Postchemoradiation intraoperative electron-beam radiation therapy boost in resected locally advanced rectal cancer: long-term results focused on topographic pattern of locoregional relapse. Radiother Oncol 112:52-58

78. Stoeckle E, Coindre JM, Bonvalot S et al (2001) Prognostic factors in retroperitoneal sarcoma: a multivariate analysis of a series of 165 patients of the French Cancer Center Federation Sarcoma Group. Cancer 92:359-368

79. Stucky CC, WasifN, Ashman JB et al (2014) Excellent local control with preoperative radiation therapy, surgical resection, and intra-operative electron radiation therapy for retroperitoneal sarcoma. JSurg Oncol 109:798-803

80. Valentini V, Calvo F, Reni M et al (2009) Intraoperative radiotherapy (IORT) in pancreaticcancer: joint analysis of the ISIORT-Europe experience. Radiother Oncol 91:54-59

81. Valentini V, Coco C, Rizzo G et al (2009) Outcomes of clinical T4M0 extra-peritoneal rectal cancer treated with preoperative radiochemotherapy and surgery: a prospective evaluation of a single institutional experience. Surgery 145:486-494

82. Valentini V, Morganti AG, De Franco A et al (1999) Chemoradiation with or without intraoperative radiation therapy in patients with locally recurrent 


\section{Übersichten}

rectal carcinoma: prognostic factors and long term outcome. Cancer 86:2612-2624

83. Vujaskovic Z, Willett CG, Tepper JE et al (2011) Normal-tissue tolerance to IOERT, EBRT, or both: animal and clinical studies. In: Gunderson LL, Willett CG, Calvo FA, Harrison LB (Hrsg) Intraoperative Irradiation: Techniques and Results. Humana, Totowa, S119-138

84. Wang LB, McAneny D, Doherty G et al (2017) Effect of intraoperative radiotherapy in the treatment of retroperitoneal sarcoma. Int J Clin Oncol 22:563-568

85. Wasserberg N (2014) Interval to surgery after neoadjuvant treatment for colorectal cancer. World J Gastroenterol 20:4256-4262

86. Wattenberg MM, Fahim A, Ahmed MM et al (2014) Unlocking the combination: potentiation of radiation-induced antitumor responses with immunotherapy. Radiat Res 182:126-138

87. Wattenberg MM, Kwilas AR, Gameiro SR et al (2014) Expanding the use of monoclonal antibody therapy of cancer by using ionising radiation to upregulate antibody targets. Br J Cancer 110:1472-1480

88. Wiig JN, Tveit KM, Poulsen JP et al (2002) Preoperative irradiation and surgery for recurrent rectal cancer. Will intraoperative radiotherapy (IORT) be of additional benefit? A prospective study. Radiother Oncol 62:207-213

89. Wing K, Onishi Y, Prieto-Martin Petal (2008) CTLA-4 control over Foxp3+ regulatory $T$ cell function. Science 322:271-275

90. Yu WW, Guo YM, Zhang Q et al (2015) Benefits from adjuvant intraoperative radiotherapy treatment for gastric cancer: a meta-analysis. Mol Clin Oncol 3:185-189

91. Zhang Q, Tey J, Peng L et al (2012) Adjuvant chemoradiotherapy with or without intraoperative radiotherapy for the treatment of resectable locally advanced gastric adenocarcinoma. Radiother Oncol 102:51-55

92. Zhang Q, Tey J, Yang Z et al (2015) Adjuvant chemoradiation plus intraoperative radiotherapy versus adjuvant chemoradiation alone in patients with locally advanced rectal cancer. Am JClin Oncol 38:11-16

\section{Hier steht eine Anzeige.}

\section{䌼 Springer}

